\title{
Undergraduate student construction and interpretation of graphs in physics lab activities
}

\author{
Ryan S. Nixon, ${ }^{1, *}$ T. J. Godfrey, ${ }^{2}$ Nicholas T. Mayhew, ${ }^{2}$ and Craig C. Wiegert ${ }^{2}$ \\ ${ }^{1}$ Department of Mathematics and Science Education, \\ University of Georgia, Athens, Georgia 30602-2451, USA \\ ${ }^{2}$ Department of Physics and Astronomy, University of Georgia, Athens, Georgia 30602-2451, USA
}

(Received 12 December 2014; published 16 February 2016)

\begin{abstract}
Lab activities are an important element of an undergraduate physics course. In these lab activities, students construct and interpret graphs in order to connect the procedures of the lab with an understanding of the related physics concepts. This study investigated undergraduate students' construction and interpretation of graphs with best-fit lines in the context of two physics lab activities. Students' graphs were evaluated for overall graph quality and for the quality of the best-fit line. The strategies students used and their understanding of the meaning of the graph were accessed through interviews. The results suggest that undergraduate introductory physics students can successfully construct graphs with best-fit lines while not connecting the meaning of the graph to the underlying physics concepts. Furthermore, results indicated that the most challenging aspect of constructing a graph is setting up the scale, and that graphing is situated in specific contexts.
\end{abstract}

DOI: 10.1103/PhysRevPhysEducRes.12.010104

\section{INTRODUCTION}

Lab activities are an "essential part" of the undergraduate physics curriculum [1]. Two themes pervade the six goals of undergraduate labs articulated by the American Association of Physics Teachers: "thinking like a physicist" and "constructing knowledge" [1]. By participating in lab activities, students ideally have opportunities to think like a physicist as they design and conduct experiments, analyze data, and communicate claims about the phenomena they have observed. This prepares students to productively participate in physics in the future and to understand the strengths and limitations of physics knowledge that has been generated in the past. Lab activities also help students construct their own physics knowledge by collecting, analyzing, and interpreting data such that they can "draw meaningful conclusions from personal observations of the physical world" [1]. By participating in lab activities, students can come to better understand the physics concepts learned outside of the lab [2,3].

Graphing, the act of interpreting and constructing graphs, plays an important role in achieving these two broad goals of lab activities. For one, graphing is a part of thinking like a physicist. Physicists use graphs to analyze and interpret data $[1,4]$. Graphs can draw out relationships

\footnotetext{
*Corresponding author.

rynixon@byu.edu

Present address: 206R MCKB, Brigham Young University, Provo, UT 84601, USA.

Published by the American Physical Society under the terms of the Creative Commons Attribution 3.0 License. Further distribution of this work must maintain attribution to the author $(s)$ and the published article's title, journal citation, and DOI.
}

and trends otherwise not visible in raw data. Graphing also help students think like physicists because graphs are an important part of scientific communication $[4,5]$. Physicists construct graphs to communicate their ideas. Students should be able to construct graphs in alignment with the norms of the discipline [1]. Physicists likewise interpret graphs constructed by others in order to evaluate the strength of their claims. To participate in physics and be a critical consumer of scientific knowledge, students need to be able to understand the message communicated by graphs. These two aspects of thinking like a physicist are highlighted in university physics recommendations and $\mathrm{K}-12$ science education standards [1,4].

When students use graphs to make sense of the data gathered in lab activities, they are assisted in developing knowledge of physics concepts, the second broad goal of physics lab activities. Physics educators often refer to the importance of labs in helping students connect theory and practice, or models with observations [6-8]. Presenting data in graphs and other representations facilitates this important link between concepts and lab activities.

Notwithstanding the importance of graphing, research has shown that students struggle with the construction and interpretation of graphs in a physics context [9-13]. Most of this research, in both physics education and mathematics education, has focused on challenges with interpreting graphs constructed by others. Other studies, though fewer in number, have shown that students experience uncertainty and difficulty while constructing graphs $[12,14]$. These studies have found errors in students' completed graphs, but have not investigated strategies students use that may contribute to these errors. A component that students regularly struggle with is creating a best-fit line and interpreting the slope of a graphed line $[12,15]$. 
In order to help students more fully understand the important practice of graphing, instructors need to recognize the strategies students use and difficulties students encounter while constructing and interpreting graphs [16-19]. The present study seeks to understand this critical and difficult juncture in undergraduate physics labs. By investigating student graph construction and interpretation in physics lab activities, this study seeks to answer the following research questions:

(1) What was the quality of students' graphs and bestfit lines?

(2) What strategies did students use to construct best-fit lines?

(3) How do students understand the meaning of bestfit lines?

(4) Does graphing performance transfer between lab activities?

\section{LITERATURE REVIEW}

Graphing includes the related processes of interpreting and constructing graphs. Two of the most commonly cited papers on graphing in physics investigated students' difficulties when interpreting kinematics graphs. McDermott, Rosenquist, and van Zee [9] investigated college and high school students' difficulties in graph interpretation and found that difficulties could be separated into two categories: difficulties connecting graphs to physical concepts and difficulties connecting graphs to real world phenomena. Difficulties with connecting graphs to physics concepts included knowing whether to extract information from the slope or the height (known as slopeheight confusion), relating graphs to each other, matching a narrative with a graph, and interpreting area under the curve. The challenges with connecting graphs to real world phenomena included difficulties in understanding graphical representations of continuous motion, constant acceleration, and negative velocity. Additionally, students struggled to separate the shape of the curve from the path of motion, an error commonly called the "graph as picture" error.

In a similar study, Beichner [10] developed a multiplechoice assessment for high school and college physics students. Though students had already received instruction on graphing, the mean student score was a meager $40 \%$. Through analyzing student responses, Beichner identified six common errors in interpreting these kinematics graphs. First, students interpreted the shape of the line as the shape of the motion of the object (graph as picture error). Second, students used the height of the line to respond to questions requesting the slope (slope-height confusion). Additionally, students did not draw appropriate distinctions between variables such as velocity and acceleration, neglecting changes that should occur in the line when these variables were changed. Students also struggled to find the slope of lines that did not pass through the origin and confused area, height, and slope. Lastly, students struggled to interpret the meaning of area under the line.

The errors found in these two seminal pieces have been found in other studies of graph interpretation. Particularly prevalent are slope-height confusion [11,18,20,21] and graph as picture error $[11,12,18,21]$. Most of these studies involved asking students to interpret graphs for which they had no context. However, interventions that provided students context for interpreting the graph, such as using computer software to graph data in real-time, have been shown to decrease common student errors [22].

Less research has been conducted regarding student abilities in constructing graphs $[12,13]$. This research found that students experience considerable difficulties when constructing graphs $[12,23]$. One study determined that while students had conceptions that aided them in graph construction, they also held many alternative conceptions that hindered their graphing performance [17]. These included graphing the data set as a single point and using multiple graphs to represent the same data set. These alternative conceptions were found to be consistent across four different graphing activities. Studies have also shown that students' abilities with constructing graphs improved with age [24] and that graph construction proficiency is linked with cognitive ability [25].

A detailed study of student graph construction reported the frequency of errors made by 93 high school student participants while constructing a graph [12]. All of these students made at least one error. The most common error related to creating the best-fit line. In general, this study found that students' "facility with graphs was generally superficial, grounded on a few, simplistic algorithms such as plotting data points. Many of them did not clearly understand either the function of graphs or their syntax [12]."

In this study, Brasell and Rowe [12] acknowledged that the "correctness" of a graph is based on the data used and the purpose of the graph. However, participants in their study were given decontextualized data to graph. Though a common strategy for evaluating student graphing performance $[2,12]$, it is problematic for two reasons. First, students may not be aware of how the data were collected or the purposes in collecting that data. This is likely to encourage the graph construction to be procedural and removed from connections with the physics concepts. Second, this practice is not congruent with either the practice of actual scientists or classroom practice. Scientists generally collect or generate their own data or, when they use communal data, the scientists are familiar with the methods of data collection and have their own purposes for analyzing the data. In lab activities, students collect their own data to graph. These data are related to concepts learned elsewhere in the course, with the lab activity designed to build the students' physics knowledge. Thus, lab activities allow students to interpret graphs in 
context and provide an appropriate situation in which to evaluate students' graphing abilities.

The majority of studies on graphing have focused on graphing in the context of kinematics [13]. Though kinematics is a foundational topic, it is only one of many topics for which graphing can be useful. Considering the important role of graphing as a practice of physicists, other topics should be investigated.

In short, extensive research indicates that students have many difficulties interpreting graphs and less abundant research suggests there are similar challenges with graph construction. This study seeks to explore students' construction of graphs from data they gathered and to examine their interpretation of the graph. Rather than focusing on a specific physics topic this study focuses on the best-fit line feature, which is both challenging and common [12,15,23].

This study approaches knowledge from a situated cognition perspective [26]. This is similar to the perspective described in Leinhardt et al. [13] and Forster [23]. In this perspective, knowledge is connected to the situations in which it is constructed and used. As such, knowledge of graphing in one domain may be different than knowledge of graphing in another domain. In their seminal paper on situated cognition, Brown, Collins, and Duguid [26] clarified that, because of the situated nature of knowledge, social context dramatically influences what is learned and known. This perspective underscores the importance of undergraduate students learning physics concepts through lab activities. Lab activities provide undergraduate students the closest approximation of the work of physicists available in an introductory physics course. Similarly, this perspective suggests that graphing ability in one context may not transfer to graphing ability in another context. This has been shown between disciplines (e.g., mathematics and physics) $[2,11,20]$, but has not been investigated between physics topics.

\section{METHODS}

This study occurred in the context of an undergraduate physics lab course at a major university in the southeastern United States. Students enrolled in this course were concurrently enrolled in a lecture course. The combined lecture and lab were the first of a two-part introductory physics sequence open to all majors. Separate introductory physics courses were offered for physics majors and engineering majors. This study includes data from participants enrolled in two parallel sections of this lab course (40 students in one, 41 students in the other). Instructors for these sections are co-authors of this paper.

\section{A. Participants}

All of the 81 students enrolled in these sections were invited to participate in the study. Thirty-two of these students (40\%) consented. All 32 provided consent for researchers to analyze the graphs they constructed as a part of their normal lab activities. Because of absences, 27 students provided data for both Lab 1 and Lab 2. This sample of 32 students will be referred to as the consented sample. Half of the students from the consented sample also comprise the interviewed sample. These 16 students agreed to participate in an interview while they were graphing during two lab activities.

Because the focus of this study is to investigate the phenomenon of graphing in lab activities (e.g., investigating the quality of students' graphs) rather than establishing relationships between variables, this size of sample is appropriate. Other studies in physics education research with similar purposes have used comparable sample sizes $[16,27]$. This sample size allows researchers to conduct meaningful quantitative analysis, while also conducting more in-depth qualitative analysis. However, as with all samples, there is the possibility for a biased sample to affect our understanding of this phenomenon.

In order to account for bias, we compared the demographic information of the whole class, the consented sample, and the interviewed sample (see Table I). This comparison indicates that both the consented and interviewed samples are comparable to the whole class in terms of major, gender, ethnicity, and class standing (see Appendix A).

It is possible that those who were interviewed were higher achieving students than those who consented to participate in the study. To check for this bias, we compared

TABLE I. Comparison of demographics for groups of students.

\begin{tabular}{|c|c|c|c|}
\hline & $\begin{array}{l}\text { All students } \\
\quad(n=81)\end{array}$ & $\begin{array}{c}\text { Consented } \\
\text { sample }(n=32)\end{array}$ & $\begin{array}{c}\text { Interviewed } \\
\text { sample }(n=16)^{\mathrm{a}}\end{array}$ \\
\hline \multicolumn{4}{|l|}{ 1. Major } \\
\hline $\begin{array}{l}\text { a. Life } \\
\text { Sciences }\end{array}$ & $86 \%$ & $94 \%$ & $100 \%$ \\
\hline $\begin{array}{l}\text { b. Other } \\
\text { science }\end{array}$ & $6 \%$ & $6 \%$ & $0 \%$ \\
\hline c. Other & $7 \%$ & $0 \%$ & $0 \%$ \\
\hline \multicolumn{4}{|l|}{ 2. Gender } \\
\hline a. Female & $73 \%$ & $59 \%$ & $44 \%$ \\
\hline b. Male & $27 \%$ & $41 \%$ & $56 \%$ \\
\hline \multicolumn{4}{|l|}{ 3. Race } \\
\hline $\begin{array}{l}\text { a. African } \\
\text { American }\end{array}$ & $12 \%$ & $13 \%$ & $13 \%$ \\
\hline $\begin{array}{l}\text { b. Caucasian/ } \\
\text { White }\end{array}$ & $62 \%$ & $72 \%$ & $69 \%$ \\
\hline c. Other & $26 \%$ & $16 \%$ & $18 \%$ \\
\hline \multicolumn{4}{|l|}{$\begin{array}{l}\text { 4. Class } \\
\text { standing }\end{array}$} \\
\hline a. Freshman & $4 \%$ & $0 \%$ & $0 \%$ \\
\hline b. Sophomore & $42 \%$ & $25 \%$ & $44 \%$ \\
\hline c. Junior & $40 \%$ & $53 \%$ & $38 \%$ \\
\hline d. Senior & $15 \%$ & $22 \%$ & $6 \%$ \\
\hline
\end{tabular}

${ }^{\mathrm{a} O n e}$ student did not report their major and class standing. 
the scores (those designed for this study, explained in the following section) of students who were interviewed with those who consented to participate but were not interviewed (see Appendix A). We found no significant differences between these two groups of students. From these analyses of bias, we conclude that although the sample was self-selected, they adequately represented the experience of the class.

In the sections that follow, participants will be identified anonymously by an identification number followed by a number indicating the associated lab activity (e.g., a quote from student 14 during Lab 2 will be cited as 14.2). This protects the anonymity of participants, as approved by our institution's Institutional Review Board for the protection of human subjects.

\section{B. Lab activities}

In order to investigate the situated nature of graphing performance, data were collected from two lab activities. As the lab activities were separated by two weeks, significant differences in graphing performance was not expected to be a result of student learning. Lab activities were selected because of the inclusion of a best-fit line. Of the ten total lab activities throughout the semester, five included a best-fit line. One instructor (the second author) provided instruction for each lab activity.

The first lab activity (called Lab 1) investigated standing waves with harmonic numbers $n=2$ through 8 . A string approximately $1 \mathrm{~m}$ in length was fixed on both ends-one end being attached to an electronic vibrating mechanism with a given frequency of $120 \mathrm{~Hz}$. Students were able to change the tension within the string by adding masses to the end of the string not attached to the vibrating mechanism. By adjusting the tension in the string, students generated various standing waves. The students recorded the mass needed to generate each standing wave and used a meter stick to measure the distance between two consecutive nodes. With this information, students calculated the tension throughout the sting, which was assumed uniform and equal to the weight of the masses added to one end, and the wave speed through the string. Constructing a graph that compared the tension $F_{T}$ with the wave speed $c$ squared afforded students the opportunity to extract the mass per unit length $\mu$ of the string based on

$$
F_{T}=\mu c^{2} .
$$

This value was obtained by calculating the slope of the best-fit line and was compared to a known value of $0.00045 \mathrm{~kg} / \mathrm{m}$.

The second lab activity (referred to as Lab 2) explored rotational motion using energy conservation principles. Students were instructed to wrap a rope of negligible mass around a cylindrical system that was supported on a horizontal axis; at the other end of the rope hung a mass.
Releasing the mass caused the cylindrical system to rotate while the hanging mass descended to the floor. The task was to find the final velocity $v$ of the hanging mass and the angular velocity $\omega$ of the cylinder at the moment the mass struck the floor. Variables measured and recorded by each student group included the following: mass $m_{1}$ added to the end of the rope, the height $h$ from which the hanging mass was released relative to the floor, time of descent (measured with a stop watch), and the radii of the rotating system. By plotting the final translational kinetic energy subtracted from the initial potential energy against the angular velocity of the rotating system squared students produced a linear graph. This relationship is expressed as

$$
m_{1} g h-\frac{1}{2} I \omega^{2}+W_{f} .
$$

No slipping between the rope and cylindrical system was assumed; therefore, the final velocity $v$ of the hanging mass was easily related to the angular speed $\omega$ for the rotating cylindrical system. To account for frictional forces acting within the experimental setup, the term $W_{f}$ represented the work done against all frictional forces, hence the positive sign. Once the previously described graph was constructed, the experimental total moment of inertia for the system could be derived from the slope of the best-fit line. The experimental moment of inertia for the system was then compared to the theoretical moment of inertia.

While these lab activities included specific step-by-step instructions for procedures in general, there were limited instructions regarding graph construction. In Lab 1, the instructions were, "Draw the best straight line possible through the data points." The graphing instructions for Lab 2 directed students to "Graph $F_{T}$ vs. $c^{2}$ and find the slope."

\section{Data sources}

Data were collected during typical class time and activities. All 32 students (consented sample) participated in the lab activity and assignment as usual, collecting, recording, and calculating data with peers. Participants handed in their graphs during class as they would normally. The graphs of students who consented to participate in the study were scanned, anonymized, and returned to the students.

Those who agreed to participate in the interview (interviewed sample) deviated from this normal pattern only during the graphing portion. When these participants reached the graphing section, they paused and brought their lab worksheet and data to an interviewer stationed in the classroom. Once with an interviewer, the student proceeded to construct the graph explaining aloud what they were thinking. Occasionally, interviewers had the student explain something they had omitted or asked the student to clarify a decision he or she had made. Once the graph was complete, students were asked four 
questions regarding their construction and interpretation of the graph and best-fit line (see Appendix B). These interviews were semi-structured, allowing for adaptation during the interview and to follow up to better understand students' reasoning. Each interview lasted approximately 20 min and took place within the normally scheduled class time. Interviews were conducted by multiple researchers and transcribed by the first three authors.

\section{Data analysis \\ 1. Graph construction}

Graph construction performance was evaluated in three ways. First, graphs were analyzed for their overall quality, considering specific graph features that contribute to the ability of the graph to communicate a message about the data and to be used as an analysis tool from which to extract information about the best-fit line. Second, student-generated best-fit lines were compared with an ideal best-fit line. This allowed for an evaluation of how well students constructed the best-fit line element of the graph. Third, transcripts were analyzed to determine what strategies students used to construct best-fit lines. Each of these processes is described in this section. Samples of student work are included in Appendix C.

Graph quality.-Past research has identified challenges with evaluating the quality of student-constructed graphs. What constitutes a high-quality graph depends in part on the norms of the discipline [4,12], as well as the purpose of the graph and the data being used. For some graphs, for example, it is important that the scales on the $x$ and $y$ axes are equivalent [12]. However, other graphs include data that cannot be graphed meaningfully with equivalent scales (e.g., when one variable has a large range and the other has a small range). Therefore, it is important to consider the data set being graphed and the purposes of the graph when evaluating graph quality.

To accomplish this goal, a rubric for graph quality was created based on data used to plot the graphs and considering the purposes of the graph (i.e., construct a best-fit line and calculate a value). Researchers developed the graph quality rubric through meeting with experts (three physics professors and two senior physics graduate students). In these meetings, researchers briefly explained the lab students were asked to do and invited the expert to graph data a student had collected during the lab. Experts were given the opportunity to complete the graph because it was assumed that actually creating the graph would allow them to think through what characteristics were important for that specific graph. Once they completed the graph, the researcher asked the expert to identify characteristics that made the graph they constructed high quality. Researchers reiterated that the question was not about what characteristics they would use to grade a student graph (as such decisions are pedagogical, rather than scientific). Once experts had listed characteristics of high quality graphs, they were asked to rank these characteristics. The lists of these experts were compared and compiled to create a rubric that represented the statements of the group as a whole (see Table II). Overall graph quality scores were computed by summing the subscores (points from each category such as "axes labeled").

Two researchers met together and scored eight graphs, reaching consensus and clarifying the meaning of the rubric. The remaining graphs were scored independently by both researchers with an interrater reliability of $96 \%$. Disagreements were discussed and consensus was reached to determine final scores.

Descriptive statistics were computed for the overall graph quality scores and for each subscore to provide an overview of the data. A Wilcoxon signed ranks test was used to determine whether there was a difference in overall graph quality scores for Lab 1 and Lab 2. The Wilcoxon signed ranks test is a nonparametric technique particularly suited for paired non-normal data [28]. Spearman rank correlation, a nonparametric test appropriate for samples that have not been randomly selected [28], was calculated to determine whether graph quality on Lab 1 was associated with graph quality on Lab 2 . Both nonparametric tests pair student data, linking, for example, student A's score on Lab 1 with student A's score on Lab 2. Participants without scores on both labs were excluded from this analysis.

Best-fit line quality.-The second analysis of student graph construction investigated the quality of the best-fit lines generated by students. In order to evaluate their graphing performance, rather than their data collection or computational abilities, the data each student collected were used to create a comparison best-fit line. To accomplish this, each student's data were input into Microsoft Excel. The slope of the regression line for that data was then calculated using

TABLE II. Graph quality rubric (points included in parentheses).

\begin{tabular}{lccc}
\hline \hline Axes labeled & Scale & Accurate data points & Cleanliness \\
\hline Quantity identified (2) & Numbers present (2) & All data points present (1) & Not cluttered (1) \\
Units provided (2) & Scale in logical increments (2) & Points plotted in & No excessive erasures \\
correct locations (1) & or scribbles (1) \\
Variables on correct axes (2) & Graph appropriate size (2) & & \\
\hline \hline
\end{tabular}


the built-in "SLOPE" function. This line will be referred to as the ideal line. Our analysis focused on the slope of the line, rather than the position of the line, as calculating a value from the slope was the focus of both lab activities.

Researchers calculated the slope of students' graphed best-fit lines by finding points on each graph and calculating the slope using $m=\Delta y / \Delta x$, where $m$ is the slope of the line, and $\Delta y$ and $\Delta x$ are the change in ordinate values and abscissa values, respectively. Points were selected to be as far apart as possible on the graph and to cross as closely as possible with intersecting gridlines. Plotted data points and points used by students were not used in this calculation. Therefore, this analysis is of the actual line that they created rather than their ability to calculate the slope or their ability to estimate the values of points on the line. The term graphed line will refer to this line.

In order to judge the quality of student best-fit lines, the slope of each graphed line was compared with the slope of the corresponding ideal line. The fractional error between the ideal slope and the graphed slope was utilized to compare the two lines. This was found by the following:

$$
\text { Fractional error in slope }=\left|\frac{m_{\text {ideal }}-m_{\text {graphed }}}{m_{\text {ideal }}}\right| .
$$

It should be noted that a small fractional error indicates a close match between the ideal and graphed lines. Thus, the smaller the fractional error, the more closely the student's line matched the ideal line. Because the direction of the difference is not significant the absolute value of the fractional error between the ideal slope and the graphed slope was used in all analyses.

Descriptive statistics provided an overview of the data set. A Wilcoxon signed ranks test was used to compare the fractional errors in slopes from Lab 1 with the fractional errors in slopes from Lab 2. This nonparametric paired samples test was used because of the non-normal nature of the data [28]. Additionally, a Spearman rank correlation test was used to determine the strength of the association between scores on Lab 1 and Lab 2. A strong association would suggest that performance on Lab 1 was a strong indicator of performance on Lab 2. As each of these techniques required a score from both labs, participants who did not provide data for Lab 1 and Lab 2 were excluded from this analysis.

This analysis brings several benefits lacked by previous techniques. For one, this technique sought to identify the overall quality of the graph rather than merely the frequency of errors [12,24]. Additionally, this technique uses a detailed rubric that was constructed from the concerns of experts and appropriately emphasizes those components that were most emphasized by experts [12]. Finally, this graph analysis technique brings with it the strength of separately treating overall graph quality and the quality of the best-fit line. This simple quantitative technique for comparing the ideal line with the graphed line provides an objective and specific judge of best-fit line quality.

Best-fit line construction strategies.-In order to understand students' strategies for constructing best-fit lines, qualitative data were utilized. Using transcriptions of interviews described above, we wrote brief summaries of the students' responses in each of these areas: strategies, purpose, and meaning [29]. These summaries were placed in a table and used to create descriptive codes [30]. After multiple passes through the data, the number of codes was decreased as similar patterns were recognized. The finalized descriptive codes for best-fit line construction strategies are reported in Sec. IV. A. 3.

\section{Graph interpretation}

Qualitative data were also utilized to explore student interpretation of best-fit lines. Though this analysis primarily focused on the responses to questions regarding the purpose and meaning of best-fit lines, the entire transcript was examined for evidence of student understanding. In order to explore student understanding of best-fit lines, we wrote brief summaries for each participant's responses, placed them in a table, and created descriptive codes (as was done with student strategies). These descriptive codes were then used as headings for columns in a construct table [29]. This table was filled in by returning to the transcripts again and identifying excerpts from each participant that represented those codes. Using these excerpts, common themes in student understanding were sought across individuals. The themes are reported in Sec. IV B.

\section{FINDINGS}

\section{A. Graph construction}

The three data analysis strategies portray unique information related to student success in constructing best-fit lines and the process these students used.

\section{Graph quality}

Graph quality was evaluated using the four-category rubric described previously. Descriptive statistics for both Lab 1 and Lab 2 are reported in Table III.

This analysis indicated that the mean graph quality score for Lab $1(M=13.6, n=30)$ was greater than the mean graph quality score for Lab $2(M=12.5, n=29)$. To determine whether there was a statistically significant difference in student graph quality on Lab 1 and Lab 2, a Wilcoxon signed ranks test was conducted. This test suggested that student graph quality scores on Lab 1 were significantly higher $(\alpha=0.05)$ than student graph quality scores on Lab $2(Z=-2.142, n=28, p=0.032)$.

The lowest subscore for both labs is scale $\left(M_{\mathrm{Lab} 1}=4.63\right.$, $\left.M_{\mathrm{Lab} 2}=4.62\right)$. Errors in this subscore included incorrectly sizing the graph, being inconsistent with the scale 
TABLE III. Graph quality scores.

\begin{tabular}{lcc}
\hline \hline & Lab 1 & Lab 2 \\
\hline 1. Total Graph Scores & & \\
a. Mean & 13.6 & 12.5 \\
& $(85 \%)$ & $(78 \%)$ \\
b. $n$ & 30 & 29 \\
c. Standard deviation & 2.04 & 1.66 \\
2. Means for Subscores & & \\
a. Axes & 5.67 & 4.63 \\
& $(95 \%)$ & $(77 \%)$ \\
b. Scale & 4.63 & 4.62 \\
& $(77 \%)$ & $(77 \%)$ \\
c. Data points & 1.80 & 1.97 \\
& $(90 \%)$ & $(99 \%)$ \\
d. Cleanliness & 1.53 & 1.59 \\
& $(77 \%)$ & $(80 \%)$ \\
\hline \hline
\end{tabular}

numbering, and selecting illogical increments to place on the axis. A Wilcoxon signed ranks test found that there was not a significant difference $(\alpha=0.05)$ between students' scale subscore on Lab 1 and students' scale subscore on Lab $2(Z=-0.903, n=28, p=0.366)$.

We were also interested in determining whether graph quality on Lab 1 was related with graph quality on Lab 2 . Results from Spearman's rank correlation test indicated that graph quality on Lab 1 was not significantly $(\alpha=0.05)$ associated with graph quality on Lab $2\left(r_{s}=0.047\right.$, $n=28, p=0.811$ )

\section{Best-fit line quality}

As described above, the quality of the best-fit line was evaluated by computing the fractional error between the ideal slope (calculated in Microsoft Excel using the student's data) and the graphed slope (measured from the student's graph). Descriptive statistics for each lab are reported in Table IV.

From these statistics we see that the mean fractional error was greater for Lab $2(M=4.453, n=28)$ than it was for Lab $1(M=0.1004, n=29)$. This suggests that students were able to draw the best-fit line for Lab 1 more accurately than they did for Lab 2. A Wilcoxon Signed Ranks test showed that the difference in fractional errors on the two

TABLE IV. Fractional error between ideal and graphed best-fit line slope (all data present).

\begin{tabular}{lcc}
\hline \hline & Lab 1 & Lab 2 \\
\hline Mean & 0.1004 & 4.453 \\
$N$ & 29 & 28 \\
Standard Deviation & 0.3410 & 18.9921 \\
Minimum & 0.0017 & 0.0000 \\
Maximum & 1.8516 & 98.9584 \\
Skewness (SE) & $5.189(.434)$ & $4.936(.441)$ \\
\hline \hline
\end{tabular}

labs was statistically significant at $\alpha=0.05(Z=-2.919$, $n=25, p=0.004$ ).

However, further attention to the data revealed the presence of outliers. These outliers are student graphs that exhibited extreme fractional errors as opposed to outlier data points on student graphs. This is apparent from the skewness of the distribution of fractional error for each lab $($ Lab 1 skewness $=5.189$, Lab 2 skewness $=4.936)$. Because of this, Statistical Package for Social Sciences (SPSS, IBM Corp., Armonk, NY, USA) software was used to create a boxplot for the data from each lab (see Figs. 1 and 2). From this, six student graphs were identified as outliers. Analysis from all of the data was reported above because these graphs represent the work of participants, rather than researcher error. The fractional error in slope for these six graphs ranged from 0.2972 to 98.96. These represent major deviations from the ideal line. After analyzing each outlier, the mistakes that were made were quite obvious. In several instances, the students simply plotted their data points incorrectly. In other instances, the error was due to a poorly chosen scale. Because the

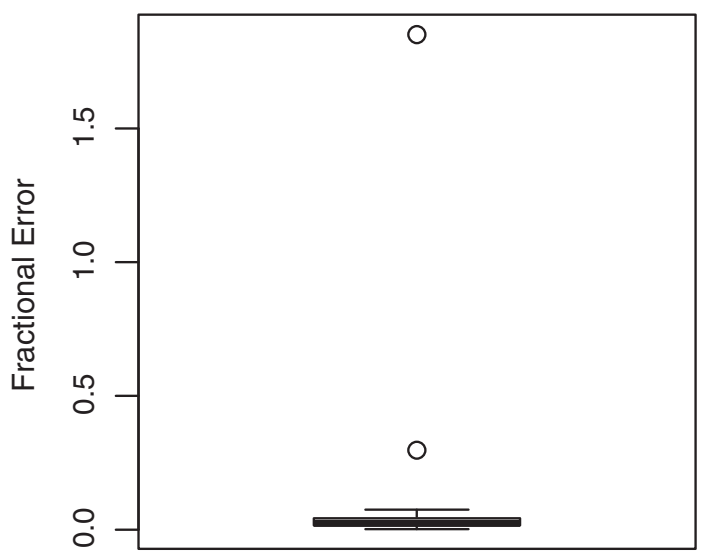

FIG. 1. Boxplot of fractional error between the ideal slope and the graphed slope for Lab 1.

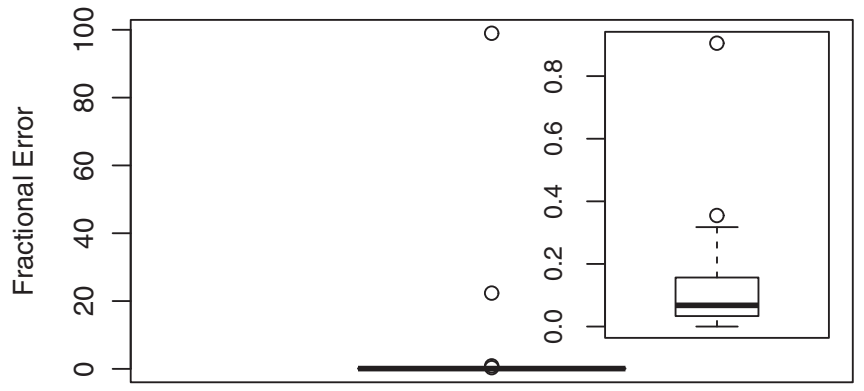

FIG. 2. Boxplot of fractional error between the ideal slope and the graphed slope for Lab 2. Note that on the larger plot there are two data points overlapping near the box at the bottom of the plot. The inset shows greater detail such that these two points can be distinguished. 
TABLE V. Fractional error between ideal and graphed best-fit line slope (outliers removed).

\begin{tabular}{lcc}
\hline \hline & Lab 1 & Lab 2 \\
\hline Mean & 0.0283 & 0.0879 \\
$N$ & 27 & 24 \\
Standard Deviation & 0.0201 & 0.0788 \\
Minimum & 0.0017 & 0.0000 \\
Maximum & 0.0744 & 0.3176 \\
Skewness (SE) & 0.874 & 1.308 \\
& $(0.448)$ & $(0.472)$ \\
\hline \hline
\end{tabular}

remainder of this analysis excluded these outliers, they are not discussed further.

For the remainder of the quantitative analysis, these six graphs were removed in order to provide a more accurate analysis of the bulk of the data. Once again, descriptive statistics were calculated (see Table V). These show that, with outliers removed, the mean fractional error in slope between the ideal line and the graphed line was 0.0283 $(n=27, \mathrm{SD}=0.0201)$ for Lab 1 and $0.0879(n=24$, $\mathrm{SD}=0.0788$ ) for Lab 2. Considering the crudeness of the methods students used to create the line, these results are rather impressive. This suggests that students, on average with outliers removed, were able to quite successfully create the best-fit line for the data that they collected.

In order to determine if there was a difference in the fractional error in ideal and graphed slopes on Lab 1 and Lab 2, a Wilcoxon signed ranks test was conducted. This test indicated that there was a significant difference $(\alpha=0.05)$ in student performance on the two labs $(Z=-2.972, \quad n=21, \quad p=0.003)$. The mean values showed that while students were quite successful (less fractional error) at constructing best-fit lines overall, students were more successful on Lab 1 than they were on Lab 2.

Finally, we were interested to see whether student performance on Lab 1 was related with student performance on Lab 2. That is, we wanted to determine whether accurately drawing a best-fit line for Lab 1 was associated with accurately drawing a best-fit line for Lab 2 . Using the data without outliers, we conducted the Spearman's rank correlation test. This statistic showed that the fractional difference in slope on Lab 1 was not associated with fractional difference in slope on Lab $2\left(r_{s}=0.008, n=20\right.$, $p=0.975, \alpha=0.05$ ). This demonstrates that accuracy on one best-fit line was not connected with accuracy on the other (see Fig. 3).

\section{Best-fit line construction strategies}

Nine strategies for best-fit line construction were identified (see Table VI). These strategies are not mutually exclusive as many of the students used more than one strategy on the same graph. We describe and provide examples of the four most common strategies.

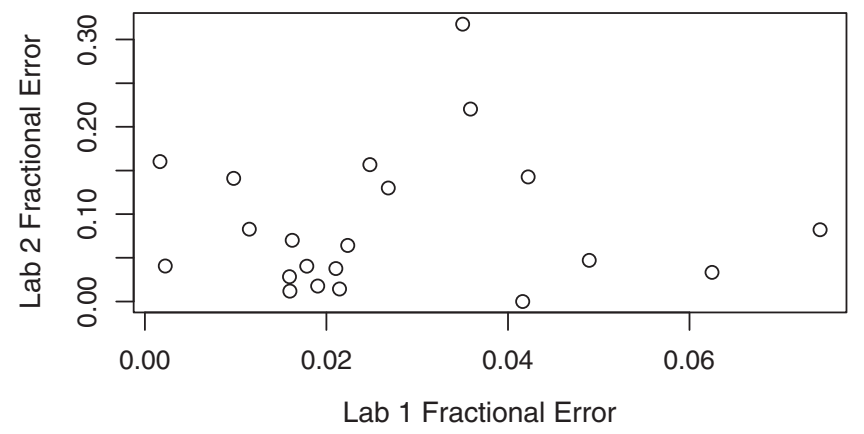

FIG. 3. Plot of Lab 1 fractional error against Lab 2 fractional error (with outliers removed).

The most common strategy students used to draw the best-fit line was to split the data in half. In this way, half of the points were above the line and half of the points were below the line. This "half and half" strategy was used in 16 instances included in this analysis with an equal amount in each lab. Students spoke of having "an even amount [of points] above and below the line" (27.1) or "the same amount of data points above the line as below" (4.1). Student 13.2 stated, "My tactic is to make sure that there's the same number of points on each side since there's five points it's not going to be exactly even but it'll be close enough." Some students used this as their initial strategy for placing the line, while others used it to check how well they had placed their line using other strategies. Though this strategy was mentioned in more instances than any other, there were only five instances in which it was used as the student's sole strategy.

Another common strategy for constructing the best-fit line was to draw a line down the middle of the data points (on 10 of 32 graphs). Rather than ensuring the same number of data points were above the line as below the line, this was a more generic strategy of estimating the central location of the data. Student 29.2 stated that you should "put your ruler in the middle of your data points and draw a line." Another student referred to this as "eyeballing" the middle of the data points (31.2). In six

TABLE VI. Instances of strategy use $(n=28)$. Note that responses were drawn from the two lab activities of 16 participants, with four participating in only one lab activity.

\begin{tabular}{lccc}
\hline \hline Strategy & Lab 1 & Lab 2 & Total \\
\hline Half and half & 8 & 8 & 16 \\
Middle of the data points & 5 & 5 & 10 \\
Get points closest to line & 4 & 1 & 5 \\
General trend of the data & 2 & 3 & 5 \\
Line up first and last points & 2 & 2 & 4 \\
Include as many points as possible & 0 & 2 & 2 \\
Start at the origin & 1 & 1 & 2 \\
Equal area above and below & 1 & 0 & 1 \\
Avoid data points & 1 & 0 & 1 \\
\hline \hline
\end{tabular}


instances this strategy was used independent of any other strategy. It was used in combination with the half and half strategy in three instances.

The last two most common strategies were used in the same number of instances ( 5 of 32 graphs each). The first of these strategies involved getting the line as close as possible to the maximum number of data points. For example, Student 25.2 explained, "I wanted to make sure most of the points that I put were relatively close to the line and that the line seemed to fit in with the points and the points didn't really seem too far from it." The second of these strategies involved drawing a line that best approximated the general trend of the data. For instance, student 31.1 said, "I'm going to try to draw a best fit line which...would be an eyeball average of all the dots combined." Student 4.1 explained that she selected the best-fit line to "show the general trend of the data."

Individual students did not use the same cluster of strategies for Lab 1 that they used on Lab 2. Of the twelve participants who described their strategies on Lab 1 and Lab 2, only two described the same process for both labs. These students relied solely on the half and half strategy. Seven of the other students reported using at least one strategy for Lab 2 that they had used on Lab 1. Three students did not use any of the same strategies on both Lab 1 and Lab 2.

\section{B. Graph interpretation}

Several themes related to student graph interpretation were found. These themes generally related to what students understood as the meaning and purpose of the best-fit line they had just constructed. In Lab 1, the best-fit line represented the relationship between the tension on the string and the wave speed squared, allowing them to calculate the mass per unit length of the string. The best-fit line in Lab 2 represented the relationship between energy (more specifically, the final kinetic energy subtracted from the initial potential energy in the system) and the angular velocity squared, allowing the calculation of the moment of inertia. In both cases, using the relationship of two variables allowed for the calculation of a characteristic of the system being studied.

\section{Students understood that best-fit lines are used to mitigate error}

Throughout the transcripts it was evident that students saw a connection between best-fit lines and mitigating error. Best-fit lines, according to many of these students, are a way to remove human error inherent in the data collection and graphing process. For example, student 26.1 explained that the purpose of a best-fit line was to "find an overall trend that you have [because] obviously with each individual calculation there may be experimental error." Student 28.2 similarly stated that a best-fit line is "basically taking an average of all the data points, so you can just kind of eliminate error."
The idea that best-fit lines remove error by "averaging" the points was quite common among this sample of students; however, there were significant variations as to how this supposed averaging was achieved. Students most commonly stated that creating a best-fit line involved finding "the average slope between all of the data points" (13.1). Some students claimed that, instead of drawing a best-fit line, one could numerically average the data and reach the same result. "The line of best fit," one student explained, "is just kind of visually for me the best way to do it" (28.2).

Another way that best-fit lines help mitigate error is by providing a means for students to check their work. Almost all students acknowledged that the best-fit line allowed them to calculate a value. While responses regarding this value varied significantly, many students indicated that this value could be compared to a known value. The level of agreement with this known value indicated how well they had performed their experiment, calculations, and graphing. Student 13.1, for example, stated that the purpose of creating a best-fit line was to check "how close you are" to the known value.

\section{Students understood that a best-fit line allowed them to calculate a value}

It was apparent from student responses that they understood best-fit lines allowed them to calculate a value. This is not surprising since each student calculated the slope of the best-fit line during the interview. While some students' remarks generally focused on the slope, others indicated what the slope meant (specifically, $\mu$ for Lab 1 and half of the moment of inertia for Lab 2). For example, student 28.1 stayed general with his discussion of the slope ("usually it's used to determine some constant, a ratio between the two factors") while student 4.1 identified the slope of the bestfit line as the "mass per unit length" of the string. Several students struggled to identify specifically what the slope stood for or did so incorrectly.

Another interesting variation in using the best-fit line to calculate values was mentioned by three students. These students suggested that a best-fit line could be used to interpolate or extrapolate other data points. Drawing a bestfit line, student 27.2 stated, is "a good simulation of multiple trials." Another student explained that with a best-fit line it is as "if you had a million more data points, it's estimating what they would look like over time" (29.2). While these statements are incorrect, they show further evidence that students understood that a best-fit line can be used to calculate a value.

\section{Students understood that best-fit lines showed connections between variables}

Almost all students demonstrated an understanding that a best-fit line showed a connection between the variables. Students used the terms trend, relationship, and correlation 
to talk about this connection. For some, this connection was general. Student 13.1 stated that a best-fit line "tells you the general trend of the different data points." Similarly, Student 29.1 explained, "A best fit means it is the line that is best trying to describe the trend that your graph is explaining."

Many students specifically commented on the observed trend of the data. For both of these graphs, the variables show a positive, linear relationship. As such, student 22.1 clarified that a best-fit line told him "the tighter the string is the more frequency you're going to have." Or, as student 17.1 stated, "if the c-squared increased then the force increases. And if the force increases, then the c-squared increases." Many of the students, however, did not specifically identify the variables involved in this relationship (e.g., "So as this would increase, this increases; as this decreases, this decreases." 12.2).

Though students understood that a best-fit line showed a connection between variables, their understanding did not seem to link to the underlying physics concepts or theory. Rather, the majority of students only had a basic, general understanding of the variables. For example, student 17.1's response did not demonstrate a thorough understanding of the variables themselves and especially did not demonstrate that the student understood the connection to the underlying theory. Additionally, statements such as the one mentioned above by student 22.1 demonstrated a misunderstanding of the concepts involved. This student claimed that the graph indicated that the frequency would increase as the tension of the string increased. While this corresponds with a simple understanding of a positive linear relationship between variables, it is not scientifically accurate. Regardless of the tension of the string, the frequency remains constant as the wave generator continues to move at a regular rate. Thus, although students understood that a best-fit line demonstrated a relationship between the variables, many of them did not understand the variables or the nature of that relationship.

Only two students provided evidence of considering best-fit lines in terms of the physics concepts. Student 19.2 indicated that the best-fit line helped her calculate the moment of inertia, which can then be compared to a known value. Not only did she identify the value calculated by the slope, she defined the moment of inertia conceptually. The other student, student 25.2, used an understanding of physics concepts to guide her graph construction and interpretation. Throughout the process of graphing she referred to the concept of energy, deciding that the best-fit line should not go through the origin because of energy "lost" due to friction. Additionally, she stated that the bestfit line enabled her to calculate the moment of inertia and the work done against friction, which is represented graphically as the $y$ intercept. These two students were unique in considering the meaning of the best-fit line in terms of the physics concepts.

\section{Student understanding of best-fit lines is context dependent}

The final theme in this section comes from an analysis of participants' responses in both labs. This analysis indicated that only 5 of 16 students demonstrated a similar understanding of best-fit lines in Lab 1 and Lab 2. The rest of the students' responses varied between the two labs. This suggested that their understanding of the meaning of best-fit lines depends on the lab activity.

\section{DISCUSSION}

This study was guided by four questions regarding the construction and interpretation of graphs involving best-fit lines in the context of two undergraduate physics lab activities. Using the above data, we answer these questions and connect the answers to the relevant literature.

A. Question 1: What was the quality of students' graphs and best-fit lines?

Overall, students produced high quality graphs and bestfit lines. This is seen in the average graph quality scores: 13.6 for Lab 1 and 12.5 for Lab 2 (out of 16). Though there is room for improvement, this suggests that the participants were not making major errors or encountering significant challenges. A mean fractional error between the ideal and graphed slope of 2.8\% for Lab 1 and $8.8 \%$ for Lab 2 (with outliers removed) corroborates this assertion. Though the lines could have been more exact, they were impressively close for the crudeness of the tools available to the students. Mistakes that were made were often related to scale construction (lowest subscore and a common error in outlier graphs).

This finding contradicts what has been found in previous studies [11-13,17,23]. As described above, previous studies reported that students experienced major procedural difficulties when constructing graphs. One explanation for this difference could be the difference in age. Participants in previous studies were primarily high school students. Wavering [24] has shown that students' success in constructing graphs improved with the increase in logical reasoning abilities that naturally accompany development. As the participants in this study were all college students, it is possible that the difficulties in graph construction experienced by high school students are no longer relevant for undergraduate students. It is also plausible that these participants, many of whom were science majors, had experienced more instruction regarding graphing than high school students.

Additionally, unlike the majority of studies on graph construction, participants in this study graphed data for which they had a context. Students had collected the data and were constructing the graph for a purpose beyond data collection for educational research. This may have mitigated some of the difficulties observed in previous research.

B. Question 2. What strategies did students use to construct best-fit lines? 
The second research question is about the strategies used by students to construct best-fit lines. From our search of the literature, no research has been published regarding this question. This study shows that most students used multiple strategies to construct best-fit lines and were not consistent with their use of strategies across the two lab activities. Almost all of these students used the half and half strategy while constructing the best-fit line, but only five used it as their sole strategy. It is interesting to note that the instructors provided this strategy to students as a rule-ofthumb for determining the general placement of the best-fit line. Though it is not certain whether students learned this strategy from the current instructors, it seems clear that this technique was taken up widely.

Most notable among the strategies students described is the reliance on rote procedures and rules of thumb, rather than an understanding of best-fit lines. It would be ideal for students to use their understanding that a best-fit line is meant to be located at the position that minimizes the distance between the line and each of the data points. Only five students referred to this idea. The remaining students drew best-fit lines without mentioning the mathematical purpose and meaning of the line.

Additionally, very few students based their strategies on an understanding of the physics concepts underlying the lab. If students approached the construction of their graph with the underlying physics in mind, one would anticipate hearing students talk about things such as whether they expected a linear fit with this equation or expected the line to pass through the origin. Only two students' explicitly discussed these ideas even after interviewers probed students to consider other strategies that could have been used to construct the best-fit line. These considerations should, but did not seem to influence students' graph construction.

C. Question 3. How do students understand the meaning of best-fit lines?

These data indicate that this sample of undergraduate students understood best-fit lines primarily in terms of their procedural value. Students spoke of best-fit lines with respect to what they do-mitigate error, show the connection between variables, and calculate a value-rather than the physics concepts they represent.

Significantly, these findings do not highlight the major misconceptions found in past literature on graph interpretation such as slope-height confusion or the graph as picture error [13]. While it is beyond the scope of these data to claim why these misconceptions were not present in this study, there are four significant differences between this study and past studies that could contribute to this distinction. First, this study used qualitative methods to understand students' interpretation of graphs rather than multiple-choice items. As such, students had the opportunity to more fully express their understanding than on a multiple-choice assessment. Second, students in this study interpreted a graph they had constructed using data they had gathered rather than interpreting a graph constructed by someone else. This provided contextual information that could have enabled them to avoid common errors. Third, these graphs were related to waves and rotational motiontopics that have not been previously explored in the graphing literature. A common error for one topic may not be a common error for another topic. Fourth, the graphs in this study included best-fit lines. Past studies have explored a variety of graph types, including curvilinear trends $[9,10]$. Errors could be different based on the type of graph involved. While this study cannot claim which of these differences made it so that common misconceptions were not apparent, these findings do suggest important areas for future research. An additional avenue for further research would be further investigating students' understanding of best-fit lines and how conceptual understanding of best-fit lines influences graph construction and interpretation.

D. Question 4. Does graphing performance transfer between lab activities?

The body of evidence here indicates that graphing performance is not transferrable between these two labs. This is seen in both the quantitative analysis of overall graph and best-fit line quality. In both cases, the scores from Lab 1 are significantly different, and are not associated with scores on Lab 2. Likewise, students' strategies and understandings differ from Lab 1 to Lab 2.

Such findings echo the results of previous research contrasting graphing in a physics context with a mathematics or everyday context $[2,11,20]$ and are consistent with a socially situated perspective of graphing [23]. Many factors could influence the differences in students graph construction and interpretation between the two labs, including the physics concepts involved or the differences in variables plotted (e.g., the $y$-axis variable was tension in Lab 1, initial potential energy minus the final translational kinetic energy of the hanging mass in Lab 2). While these data cannot indicate what led to these differences, it is clear that student graph construction and interpretation is situated. Though studies have shown differences in graph construction and interpretation between disciplinary contexts, this is the first study to show situated differences within the physics discipline. Further research accounting for the social situations of lab activities and the knowledge situated therein are therefore appropriate.

\section{CONCLUSIONS AND IMPLICATIONS}

From these findings we draw three conclusions. Each of these conclusions is described with attendant implications.

\section{A. Students can successfully construct graphs, but generally do not consider the underlying physics}

The first conclusion from these data is that undergraduate introductory physics students can successfully construct 
graphs with best-fit lines while appearing not to connect these graphs to the underlying physics concepts. The students in this study constructed high quality graphs and best-fit lines. However, as they spoke about the meaning of the best-fit line they had just constructed, their discussions focused on the general procedural use rather than the meaning for the physics underlying the lab they had just completed.

One important implication for instruction based on this conclusion is that students need to be pushed to connect the lab activity to the physics concepts the lab activity is intended to teach. Without this emphasis, students may succeed at constructing graphs without linking these graphs to the physics concepts and fall short of enabling students to think like physicists or construct knowledge of physics concepts [1]. An examination of the lab manual and the anecdotal reports of the instructors indicated that communications to students primarily focused on procedures for completing the task. References to these procedures appear frequently in our interviews with students. This emphasis on procedures may have discouraged students from considering the underlying physics of the lab activity.

Simple modifications to lab activities that are procedurally focused could encourage students to connect the graph to the physics concepts. For example, concluding a lab activity with a series of questions that help students connect the activity with the physics ideas, rather than simply calculating the percent error or making a brief comment about how well the experimental value matched the true value. This could also be achieved through discussions occurring in the lecture portion of the course. Additionally, instructors should be explicitly aware of the conceptual purposes of the lab activities they lead and use instructions to point students to the underlying physics concepts.

This is the first research of which we are aware that investigated how students connect lab activities to physics concepts through graphing. Future research should explore more in depth how students understand graphs in general and best-fit lines specifically. Following this line of research, researchers should further investigate how lab activities and graphing influence conceptual development in physics courses. Moreover, future research should investigate the effectiveness of efforts to help students connect graphing to physics concepts. Research on graphing in physics needs also to continue to move beyond the oft-studied topic of kinematics.

\section{B. Setting up the scale is the most challenging part of graph construction}

A second conclusion that can be reached from this body of evidence is that the most challenging part of constructing a graph from a lab activity is setting up the scales. As seen above, these students were generally successful at constructing the graphs and best-fit lines. However, the lowest graph quality subscore was in the area of scale.
Additionally, an analysis of the graphs that were excluded as outliers revealed that many of these graphs (including those with the largest fractional error) incorrectly set up the scales for their graphs. Furthermore, a large portion of the interview time was filled with students setting up the scales for their graphs.

Though an important task, setting up the scales is not likely an important piece of helping students understand the underlying physics concepts related to the graph, one of the primary purposes of undergraduate physics labs [1]. It is probable that spending time creating the scales decreases time that could be spent connecting the graph to the physics concepts that constitute the aim of the lab activityespecially in lab situations where the activity must be performed, completed, and turned in during a single class meeting time. Such a procedurally focused task likely contributes to the students' focus on procedure and limited attention to the physics concepts.

In order to encourage students to focus on the physics concepts related to the graphs and best-fit lines, we recommend that instructors make efforts to limit the procedural load of constructing graphs, particularly related to setting up scales. One way to limit this procedural load would be providing more specific guidance on scale development. This instruction should emphasize the reasons underlying the decisions that must be made while setting up scales and should point students toward a deeper connection between their graphs and the physics concepts.

Lowering the procedural load of graph construction could also be accomplished by allowing students to utilize computer software to set up the scales. This would provide students greater time to interpret the graph and connect with the physics concepts being taught in the lab activity. In other contexts, scholars have argued that the nature of a task can change based on the tools being used $[31,32]$. For example, research on note taking has found that students tend to transcribe what is being said when they use a computer to take notes, rather than summarizing key points when writing notes by hand [33]. This can diminish student learning, as the task is focused on recording rather than processing information. Similarly, the findings of the current study suggest that students focus on the procedure of constructing a graph when drawing a graph by hand. Using computer software to construct a graph may allow students to focus on interpreting and understanding the meaning of the graph. The effect of using computer software to construct graphs, as opposed to hand graphing, needs to be further evaluated.

\section{Graph construction and interpretation is situated}

The third conclusion drawn from these data is that graphing is situated in specific contexts $[13,23]$. This analysis revealed differences between Lab 1 and Lab 2 in terms of graph quality, best-fit line quality, graph 
construction strategies, and student understanding of bestfit lines.

The situated nature of graphing brings with it some implications for instructors. Instructors should not solely focus their instruction during lab activities on completing the procedures to collect data for the lab. Explicit instruction regarding the construction of the graph that day may be necessary in order to help students understand the unique considerations of constructing and interpreting the activity's graph. Instructors should not assume that experience in previous mathematics or science courses will be sufficient to construct and interpret graphs in physics lab activities. Instruction based in the context of the physics concepts, rather than rote procedures and rules-of-thumb, seem likely to be most effective at helping students successfully construct and interpret graphs. Furthermore, research on student graph construction and interpretation should be conducted in the context of lab activities.

This study makes an important contribution in understanding undergraduate physics students' construction and interpretation of best-fit line graphs in physics lab activities. These activities have the potential to provide powerful learning opportunities for students. However, without changes, it is likely that students will continue to primarily learn how to follow procedures rather than physics concepts.

\section{ACKNOWLEDGMENTS}

Thanks to Julie Luft for her support and assistance throughout this project. Also, thanks to Benjamin Cooley, Shannon Dubois, Jason Gilchrist, Nick Gomez, Bryant Jensen, Steven Lewis, Loris Magnani, Mark Mudrick, Jaclyn Murray, Bahadir Namdar, Rich Ross, and Laura Schneider for their help during data collection and analysis. This project has been funded in part by the University of Georgia Department of Physics and Astronomy.

\section{APPENDIX A: ANALYSIS OF POTENTIAL BIAS OF INTERVIEWED SAMPLE}

In order to determine whether the samples represented the class as a whole, the demographics of the class as a whole were compared with both the demographics of the consented sample and the demographics of the interviewed sample. As the intent was to compare the distribution of each sample with the distribution of the entire class, the chisquared goodness of fit test was used. Results of these tests indicate that no statistically significant differences exist between the class and the two samples used in the study in terms of gender and race (see Table VII). Tests were unable to be conducted for the variables of major and class standing, since there were not participants in each category.

To further investigate potential bias in the sample, the scores of the interviewed sample participants were compared with the scores of the consented sample participants. These scores included graph quality scores and fractional
TABLE VII. Demographics comparisons of consented and interviewed sample with class using chi-squared goodness of fit tests.

\begin{tabular}{lcccccccc}
\hline \hline & \multicolumn{3}{c}{ Consented sample } & & \multicolumn{3}{c}{ Interviewed sample } \\
\cline { 1 - 3 } & $\chi 2(d f)$ & $N$ & $p$ & & $\chi^{2}(d f)$ & $N$ & $p$ \\
\hline Gender & $2.919(1)$ & 32 & 0.088 & & $7.025(1)$ & 16 & 0.008 \\
Race & $1.835(2)$ & 32 & 0.400 & & $0.417(2)$ & 16 & 0.812 \\
\hline \hline
\end{tabular}

TABLE VIII. Comparison of scores of interviewed sample with scores of those who consented without being interviewed.

\begin{tabular}{lccc}
\hline \hline & $n$ & Mann-Whitney $U$ & $p$ \\
\hline Lab 1 graph quality score & 30 & 97.5 & 0.524 \\
Lab 2 graph quality score & 29 & 78.5 & 0.234 \\
Lab 1 fractional difference & 30 & 93.0 & 0.430 \\
Lab 2 fractional difference & 28 & 94.0 & 0.854 \\
\hline \hline
\end{tabular}

errors for Lab 1 and Lab 2 (see Secs. III D 1 a and III D 1 b for further details). This analysis entailed a series of MannWhitney U tests, a nonparametric test comparable to a two sample $t$ test. The Mann-Whitney $\mathrm{U}$ test, however, is better suited for small samples that are not normally distributed, as is the case with these data.

No significant differences were observed between scores for students who consented without being interviewed and students that were interviewed (see Table VIII).

\section{APPENDIX B: INTERVIEW PROTOCOL}

In this section we provide the interview protocol used in this study.

Think-Aloud

(1) Have students explain their thinking as they work through the process of making a graph and bestfit line.

(2) Ask clarifying, nonjudgmental questions to understand their thinking while making the graph.

Interview Questions (follow up as needed):

(1) What other ways could you think of to make a bestfit line? Why did you choose this way instead of those? (Focus on the conceptual tools rather than the physical tools. Other conceptual methods for creating a best-fit line.)

(2) What makes drawing a best-fit line hard?

(3) What is the purpose of drawing a best-fit line in a physics lab?

(4) What does a best-fit line mean?

\section{APPENDIX C: SAMPLES OF STUDENT GRAPHS}

In this section, we provide four samples of student graphs. There are two graphs from each lab, one high-rated graph and one low-rated graph. 


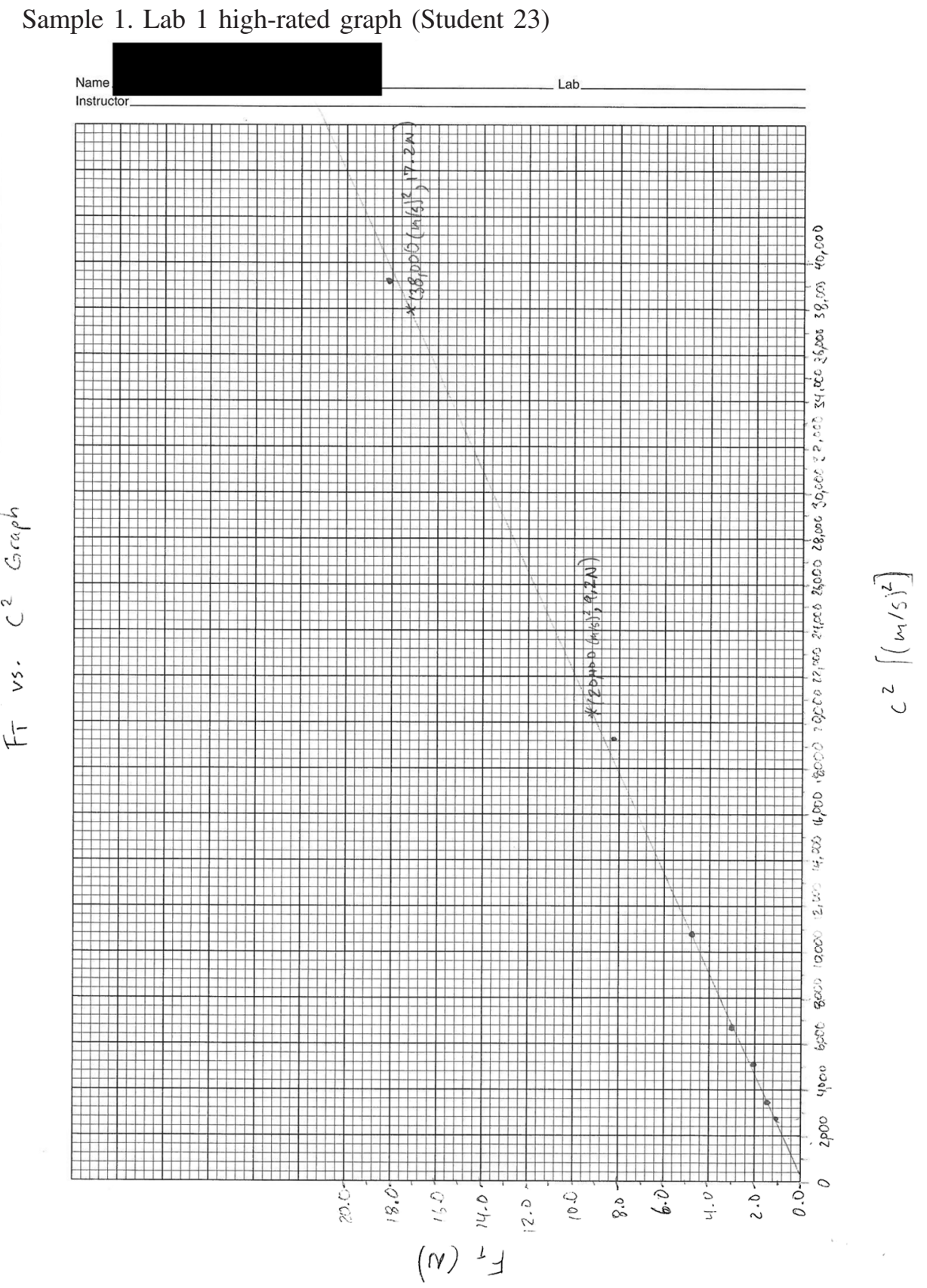

This graph was given full points for all subscores of graph quality. It also had one of the lowest fractional errors in Lab 1.

\begin{tabular}{lccccc}
\hline $\begin{array}{l}\text { Overall graph } \\
\text { quality }\end{array}$ & Axes & Scale & $\begin{array}{c}\text { Data } \\
\text { points }\end{array}$ & Cleanliness & $\begin{array}{c}\text { Fractional } \\
\text { error }\end{array}$ \\
\hline 16 & 6 & 6 & 2 & 2 & 0.0159 \\
\hline
\end{tabular}


Sample 2. Lab 1 low-rated graph (Student 12)

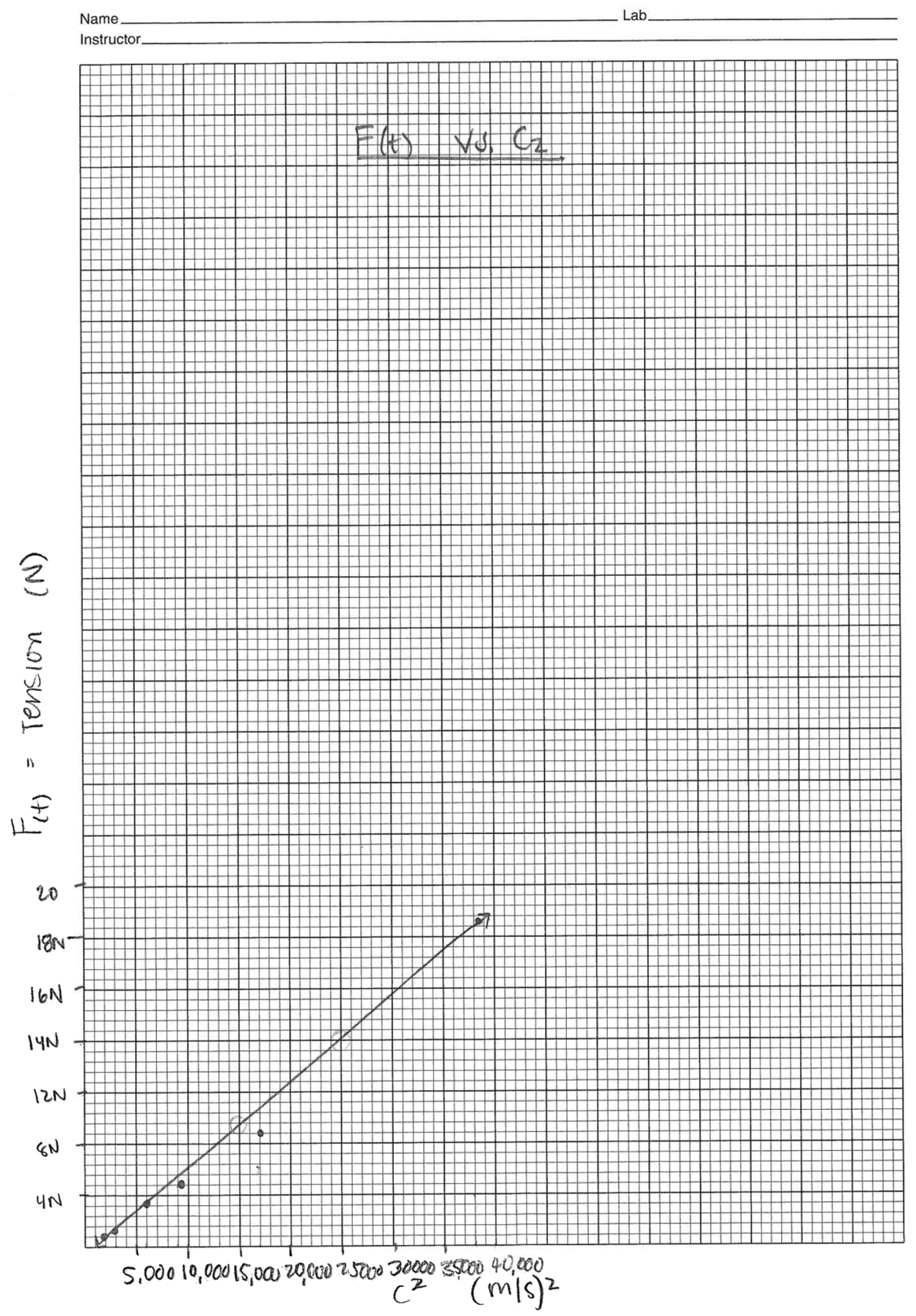

This graph received one of the lowest scores in Lab 1 for graph quality. While the axes are adequately set up, there are problems with each of the other elements. In terms of the scale, the graph does not utilize the space allowed, limiting the potential precision of the construction of the best-fit line. Additionally, the student made an error labeling the $y$ axis (started with increments of $4 \mathrm{~N}$, ended with increments of $2 \mathrm{~N}$ ). The graph was marked down on the data points subscore because one data point was not plotted. Finally, the graph was marked down on the cleanliness subscore as it contains multiple erasures still visible on the body of the graph and the axes. This graph had one of the largest fractional errors.

\begin{tabular}{lccccc}
\hline $\begin{array}{l}\text { Overall graph } \\
\text { quality }\end{array}$ & Axes & Scale & $\begin{array}{c}\text { Data } \\
\text { points }\end{array}$ & Cleanliness & $\begin{array}{c}\text { Fractional } \\
\text { error }\end{array}$ \\
\hline 11 & 6 & 2 & 1 & 1 & 0.0247 \\
\hline
\end{tabular}


Sample 3. Lab 2 high-rated graph (Student 23)



This graph received full points for all graph quality subscores and had one of the lowest fractional errors in Lab 2

\begin{tabular}{lccccc}
\hline $\begin{array}{l}\text { Overall } \\
\text { graph } \\
\text { quality }\end{array}$ & Axes & Scale & $\begin{array}{c}\text { Data } \\
\text { points }\end{array}$ & Cleanliness & $\begin{array}{c}\text { Fractional } \\
\text { error }\end{array}$ \\
\hline 16 & 6 & 6 & 2 & 2 & 0.0283 \\
\hline
\end{tabular}


Sample 4. Lab 2 low-rated graph (Student 26)

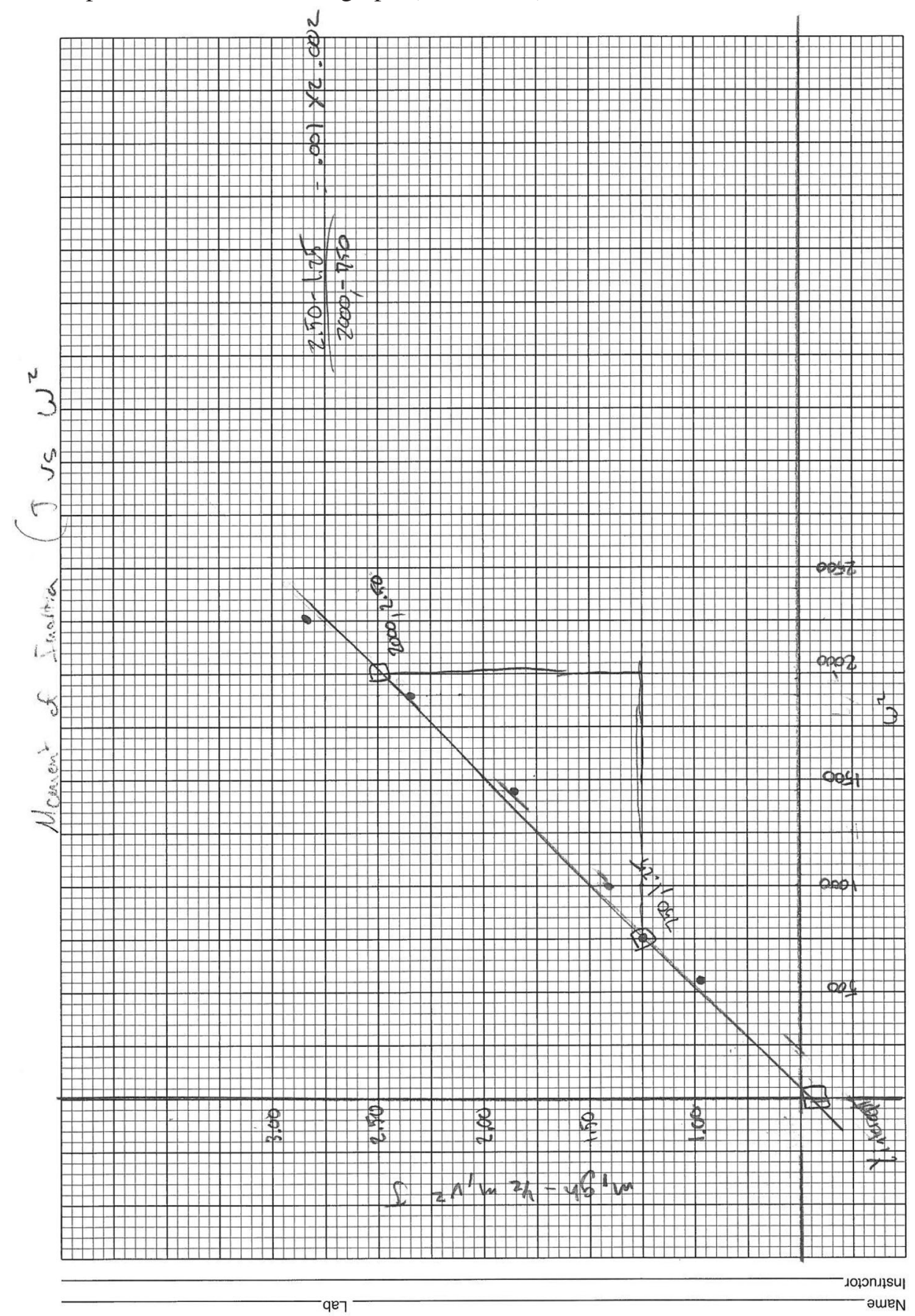


This graph received one of the lowest graph quality scores for Lab 2. Points were removed from the axes subscore for not providing the units on the $x$ axis. The scale subscore was reduced because the student did not use a consistent scale on the $y$ axis (first increment is $1 \mathrm{~J}$, all others are $0.5 \mathrm{~J}$ ) and because the graph does not fill the available space. The graph contains remnants of a previous best-fit line and multiple other markings that distract from the purpose of the graph.

\begin{tabular}{lccccc}
\hline $\begin{array}{l}\text { Overall } \\
\text { graph quality }\end{array}$ & Axes & Scale & $\begin{array}{c}\text { Data } \\
\text { points }\end{array}$ & Cleanliness & $\begin{array}{c}\text { Fractional } \\
\text { error }\end{array}$ \\
\hline 9 & 4 & 2 & 2 & 1 & 0.0379 \\
\hline
\end{tabular}

[1] American Association of Physics Teachers (AAPT), AAPT Recommendations for the Undergraduate Physics Laboratory Curriculum, 2014.

[2] J. Woolnough, How do students learn to apply their mathematical knowledge to interpret graphs in physics?, Res. Sci. Educ. 30, 259 (2000).

[3] R. D. Knight, Five Easy Lessons: Strategies for Successful Physics Teaching (Pearson Education, San Fransico, CA, 2002).

[4] National Research Council (NRC), A Framework for K-12 Science Education: Practices, Crosscutting Concepts, and Core Ideas (National Academies Press, Washington, DC, 2012).

[5] J. Lemke, in Reading Science: Critical, and Functional Perspectives on Discourses of Science, edited by J. R. Martin and R. Veel (Routledge, London, England, 1998), p. 87.

[6] National Research Council (NRC), Taking Science to School: Learning and Teaching Science in Grades K-8 (National Academies Press, Washington, DC, 2007).

[7] R. Duit, H. Schecker, D. Höttecke, and H. Niedderer, in Handbook of Research on Science Education, edited by N. G. Lederman and S. K. Abell (Routledge, New York, NY, 2014), p. 434.

[8] R. T. White, The link between the laboratory and learning, Int. J. Sci. Educ. 18, 761 (1996).

[9] L. C. McDermott, M. L. Rosenquist, and E. H. van Zee, Student difficulties in connecting graphs and physics: Examples from kinematics, Am. J. Phys. 55, 503 (1987).

[10] R. J. Beichner, Testing student interpretation of kinematics graphs, Am. J. Phys. 62, 750 (1994).

[11] M. Planinic, L. Ivanjek, A. Susac, and Z. Milin-Sipus, Comparison of university students' understanding of graphs in different contexts, Phys. Rev. ST Phys. Educ. Res. 9, 020103 (2013).

[12] H. M. Brasell and M. B. Rowe, Graphing skills among high school physics students, School Sci. Math. 93, 63 (1993).

[13] G. Leinhardt, O. Zaslavsky, and M. K. Stein, Functions, graphs, and graphing: Tasks, learning, and teaching, Rev. Educ. Res. 60, 1 (1990).

[14] E. H. van Zee and L. C. McDermott, in Misconceptions and Educational Strategies in Science and Mathematics. Proceedings of the International Seminar, edited by J. D. Novak (Cornell University, Ithaca, NY, 1987), p. 531.
[15] G. M. Bowen and W.-M. Roth, Data and graph interpretation practices among preservice science teachers, J. Res. Sci. Teach. 42, 1063 (2005).

[16] A. Maries and C. Singh, Exploring one aspect of pedagogical content knowledge of teaching assistants using the test of understanding graphs in kinematics, Phys. Rev. ST Phys. Educ. Res. 9, 020120 (2013).

[17] Z. R. K. B. Mevarech, From verbal descriptions to graphic representations: Stability and change in students' alternative conceptions, Educ. Stud. Math. 32, 229 (1997).

[18] J. R. Mokros and R. F. Tinker, The impact of microcomputer-based labs on children's ability to interpret graphs, J. Res. Sci. Teach. 24, 369 (1987).

[19] P. Shah and J. Hoeffner, Review of graph comprehension research: Implications for instruction, Educ. Psychol. Rev. 14, 47 (2002).

[20] M. Planinic, Z. Milin-Sipus, H. Katic, A. Susac, and L. Ivanjek, Comparison of student understanding of line graph slope in physics and mathematics, Int. J. Sci. Math. Educ. 10, 1393 (2012).

[21] J. Clement, J. R. Mokros, and K. Schultz, Proceedings of the Annual Meeting of the American Educational Research Association (Technical Education Research Center, Cambridge, Massachusetts, 1985).

[22] H. Brasell, The effect of real-time laboratory graphing on learning graphic representations of distance and velocity, J. Res. Sci. Teach. 24, 385 (1987).

[23] P. A. Forster, Graphing in physics: Processes and sources of error in tertiary entrance examinations in Western Australia, Res. Sci. Educ. 34, 239 (2004).

[24] M. J. Wavering, Logical reasoning necessary to make line graphs, J. Res. Sci. Teach. 26, 373 (1989).

[25] C. A. Berg and D. G. Phillips, An investigation of the relationship between logical thinking structures and the ability to construct and interpret line graphs, J. Res. Sci. Teach. 31, 323 (1994).

[26] J. S. Brown, A. Collins, and P. Duguid, Situated cognition and the culture of learning, Educ. Res. 18, 32 (1989).

[27] N. Didiş, A. Eryılmaz, and Ş. Erkoç, Investigating students' mental models about the quantization of light, energy, and angular momentum, Phys. Rev. ST Phys. Educ. Res. 10, 020127 (2014).

[28] J. J. Higgins, An Introduction to Modern Nonparametric Statistics (Brooks/Cole, Australia, 2004). 
[29] M. B. Miles, A. M. Huberman, and J. Saldaña, Qualitative Data Analysis: A Methods Sourcebook 3rd ed. (Sage, Los Angeles, CA, 2014).

[30] J. Saldaña, The Coding Manual for Qualitative Researchers 2nd ed. (Sage, Los Angeles, CA, 2013).

[31] M. Goos, P. Galbraith, and P. Renshaw, in Mathematics education: Exploring the Culture of Learning, edited by
B. Allen and S. Johnston-Wilder (Routledge, London, 2003), p. 91.

[32] E. Negueruela-Azarola, in Provo Vygotskian Collective (Provo, UT, 2015, November).

[33] M. C. Friedman, (Harvard Initiative for Learning and Teaching, Cambridge, MA, 2014). 